\title{
Transition from localized to extended eigenstates in the ensemble of power-law random banded matrices
}

\author{
Alexander D. Mirlin ${ }^{1} \dagger$, Yan V. Fyodorov ${ }^{2} \dagger$, \\ Frank-Michael Dittes ${ }^{3}$, Javier Quezada ${ }^{4}$ and Thomas H. Seligman ${ }^{5}$ \\ ${ }^{1}$ Institut für Theorie der Kondensierten Materie, Universität Karlsruhe, 76128 Karlsruhe, \\ Germany \\ ${ }^{2}$ Fachbereich Physik, Universität-GH Essen, 45117 Essen, Germany \\ ${ }^{3}$ Forschungszentrum Rossendorf, Institut für Kern- und Hadronenphysik, 01314 Dresden, \\ Germany \\ 4 Tecnologico de Monterey, Guadalajara Campus, Guadalajara, Mexico \\ ${ }^{5}$ IFUNAM Laboratorio de Cuernavaca, 62191 Cuernavaca, Morelos, Mexico
}

(February 1, 2008)

\begin{abstract}
We study statistical properties of the ensemble of large $N \times N$ random matrices whose entries $H_{i j}$ decrease in a power-law fashion $H_{i j} \sim|i-j|^{-\alpha}$. Mapping the problem onto a nonlinear $\sigma$-model with non-local interaction, we find a transition from localized to extended states at $\alpha=1$. At this critical value of $\alpha$ the system exhibits multifractality and spectral statistics intermediate between the Wigner-Dyson and Poisson one. These features are reminiscent of those typical for the mobility edge of disordered conductors. We find a continuous set of critical theories at $\alpha=1$, parametrized by the value of the coupling constant of the $\sigma$-model. At $\alpha>1$ all states are expected to be localized with integrable power-law tails. At the same time, for $1<\alpha<$ $3 / 2$ the wave packet spreading at short time scale is superdiffusive: $\langle|r|\rangle \sim$ $t^{\frac{1}{2 \alpha-1}}$, which leads to a modification of the Altshuler-Shklovskii behavior of the spectral correlation function. At $1 / 2<\alpha<1$ the statistical properties of eigenstates are similar to those in a metallic sample in $d=(\alpha-1 / 2)^{-1}$ dimensions. Finally, the region $\alpha<1 / 2$ is equivalent to the corresponding Gaussian ensemble of random matrices $(\alpha=0)$. The theoretical predictions are compared with results of numerical simulations.
\end{abstract}

PACS numbers: 71.30.+h, 71.55.Jv, 05.45.+b, 72.15.Rn 


\section{INTRODUCTION.}

Recently, there has been a considerable interest in the properties of large $N \times N$ random banded matrices (RBM). The ensemble of RBM is defined as the set of matrices with elements

$$
H_{i j}=G_{i j} a(|i-j|),
$$

where the matrix $G$ runs over the Gaussian Orthogonal Ensemble (GOE), and $a(r)$ is some function satisfying the condition $\lim _{r \rightarrow \infty} a(r)=0$ and determining the shape of the band. In the most frequently considered case of RBM the function $a(r)$ is considered to be fast (at least, exponentially) decaying when $r$ exceeds some typical value $b$ called the bandwidth. Matrices of this sort were first introduced as an attempt to describe an intermediate level statistics for Hamiltonian systems in a transitional regime between complete integrability and fully developed chaos [1] and then appeared in various contexts ranging from atomic physics (see [2] and references therein) to solid state physics [3] and especially in the course of investigations of the quantum behavior of periodically driven Hamiltonian systems [4]. The mostly studied system of the latter type is the so-called quantum kicked rotator (KR) [5] characterized by the Hamiltonian

$$
\hat{H}=\frac{\hat{l}^{2}}{2 I}+V(\theta) \sum_{m=-\infty}^{\infty} \delta(t-m T)
$$

where $\hat{l}=-i \hbar \partial / \partial \theta$ is the angular momentum operator conjugated to the angle $\theta$. The constants $T$ and $I$ are the period of kicks and the moment of inertia, correspondingly, and $V(\theta)$ is usually taken to be $V(\theta)=k \cos \theta$. Classically, the KR exhibits an unbound diffusion in the angular momentum space when the strength of kicks $k$ exceeds some critical value. It was observed, however, that in a quasi-classical regime quantum effects suppress the classical diffusion [0] in close analogy with the effect of Anderson localization of a quantum particle by a random potential [ [6].

It is natural to consider the evolution (Floquet) operator $\hat{U}$ that relates values of the wavefunction over one period of perturbation, $\psi(\theta, t+T)=\hat{U} \psi(\theta, t)$, in the "unperturbed" basis of eigenfunctions of the operator $\hat{l}:|l\rangle=\frac{1}{(2 \pi)^{1 / 2}} \exp (i n \theta), n= \pm 0, \pm 1, \ldots$. The matrix elements $\langle m|U| n\rangle$ tend to zero when $|m-n| \rightarrow \infty$. In the case $V(\theta)=k \cos \theta$ this decay is faster than exponential when $|m-n|$ exceeds $b \approx k / \hbar$, whereas within the band of the effective width $b$ matrix elements prove to be pseudorandom [5]. All these observations attracted much interest to the statistical properties of the ensemble of RBM in order to use the extracted information for understanding the dynamical properties of the KR.

Let us note, however, that the fast decay of $\langle m|U| n\rangle$ in the above mentioned situation is due to the infinite differentiability of $V(\theta)=\cos \theta$. If we took a function $V(\theta)$ having a discontinuity in a derivative of some order the corresponding matrix elements of the evolution operator would decay in a power-law fashion when $|n-m| \rightarrow \infty$. In fact, there

\footnotetext{
${ }^{1}$ The authors are grateful to F. Izrailev for attracting their attention to this fact.
} 
is an interesting example of a periodically driven system where the matrix elements of the evolution operator decay in a power-law way, namely the so-called Fermi accelerator [7]. This system does not show the effect of dynamical localization in energy space typical for the KR. One can hope to understand this difference in behavior studying properties of RBM with power-law decay of the function $a(r)$ at infinity.

One may also consider the random matrix (1) as the Hamiltonian of a one-dimensional tight-binding model with long-ranged off-diagonal disorder (random hopping). A closely related problem with non-random long-range hopping and diagonal disorder was studied numerically in Ref. [8]. The qualitative effect of weak long-range hopping on the localized states in a $3 D$ Anderson insulator was discussed by Levitov [9]. We will discuss the correspondence between our results and those of Ref. [9] later on. Let us note that similar models with a power-law hopping appear also in other physical contexts [10].

As it was shown in Ref. [11, 3] the conventional RBM model can be mapped onto a $1 D$ supermatrix non-linear $\sigma$-model, which allows for an exact analytical solution. The same $\sigma$-model was derived initially for a particle moving in a quasi- $1 D$ system (a wire) and being subject to a random potential. All states are found to be asymptotically localized, with the localization length

$$
\xi=\frac{B_{2}\left(2 B_{0}-E^{2}\right)}{8 B_{0}^{2}} \propto b^{2} ; \quad B_{k}=\sum_{r=-\infty}^{\infty} a^{2}(r) r^{k}
$$

In the present paper we consider the case of a power-like shape of the band:

$$
a(r) \propto r^{-\alpha} \quad \text { for large } r .
$$

Under these conditions, the derivation of the $\sigma$-model presented in [3, 11] loses its validity.

Reconsidering this derivation, we arrive at a more general $1 D \sigma$-model with long-range interaction. Performing its perturbative and renormalization group analysis, we find that the model is much richer than the conventional short-range one. In particular, it exhibits an Anderson localization transition at $\alpha=1$. The main scope of the present work is to study the statistical properties of the model in the whole range of the parameter $\alpha$.

\section{THE POWER-LAW RANDOM BANDED MATRIX ENSEMBLE AND THE EFFECTIVE NON-LINEAR $\sigma$-MODEL}

Let us consider the ensemble given by eq. (11) with the function $a(r)$ having the form

$$
a(r)=\left\{\begin{array}{c}
1, \quad r \leq b \\
(r / b)^{-\alpha}, \quad r>b
\end{array} .\right.
$$

The parameter $b$ will serve to label the critical models with $\alpha=1$. We will consider $b$ to be large: $b \gg 1$, in order to justify formally the derivation of the $\sigma$-model. We will argue later on that our conclusions are qualitatively valid for arbitrary $b$ as well. We will call the ensemble (1), (5) the power-law random banded matrix (PRBM) model.

In Ref. [11, 3] it was shown that the RBM model (11) can be mapped, for arbitrary bandshape $a(r)$, onto a field-theoretical model of interacting $8 \times 8$ supermatrices $\sigma_{i},(i=$ $1,2, \ldots, N)$ characterized by the action 


$$
\mathcal{S}\{\sigma\}=\operatorname{Str}\left\{\frac{1}{2} \sum_{i, j} R_{i j} \sigma_{i} \sigma_{j}+\sum_{i} U\left(\sigma_{i}\right)\right\}
$$

Here $R_{i j}=\left(A^{-1}\right)_{i j}-\frac{1}{N} \delta_{i j} \sum_{k l}\left(A^{-1}\right)_{k l} ; A$ is the matrix with elements $A_{i j}=a^{2}(|i-j|)$,

$$
U(\sigma)=\frac{1}{2} \operatorname{Str}\left\{\ln \left(E-\sigma-i \frac{\omega}{2} \Lambda\right)+\frac{1}{N} \sum_{k, l}\left(A^{-1}\right)_{k l} \sigma^{2}\right\}
$$

$\omega$ is the frequency, and $\Lambda=\operatorname{diag}(I,-I)$ in the "advanced-retarded" representation. The supermatrix $\sigma_{i}$ can be parametrized as $\sigma_{i}=T_{i}^{-1} P_{i} T_{i}$, with $P_{i}$ being block-diagonal in the "advanced-retarded" decomposition and $T_{i}$ belonging to a certain graded coset space, see the reviews 12,13. For $b \gg 1$ the integral over the matrices $P_{i}$ can be evaluated by the saddle-point method [11]. Then the action (6) is reduced to a $\sigma$-model on a lattice:

$$
\mathcal{S}\{Q\}=-\frac{1}{4}\left(\pi \nu A_{0}\right)^{2} \operatorname{Str} \sum_{i j}\left[\left(A^{-1}\right)_{i j}-A_{0}^{-1} \delta_{i j}\right] Q_{i} Q_{j}-\frac{i \pi \nu \omega}{4} \sum_{i} \operatorname{Str} Q_{i} \Lambda .
$$

Here $Q_{i}=T_{i}^{-1} \Lambda T_{i}$ satisfies the constraint $Q_{i}^{2}=1, A_{0}$ is given by $A_{0}=\sum_{l} A_{k l} \approx$ $\sum_{r=-\infty}^{\infty} a^{2}(r)$, and $\nu$ is the density of states:

$$
\nu=\frac{1}{2 \pi A_{0}}\left(4 A_{0}-E^{2}\right)^{1 / 2}
$$

The standard next step is to restrict oneself to the long wavelength fluctuations of the $Q$-field. For usual RBM characterized by a function $a(r)$ decreasing faster than any power of $r$ as $r \rightarrow \infty$, this is achieved by the momentum expansion of the first term in the action $(8)$ :

$$
\begin{aligned}
\sum_{i j}\left[\left(A^{-1}\right)_{i j}\right. & \left.-A_{0}^{-1} \delta_{i j}\right] Q_{i} Q_{j} \equiv \sum_{q}\left[A_{q}^{-1}-A_{0}^{-1}\right] Q_{q} Q_{-q} \\
& \approx \frac{B_{2}}{2 A_{0}} \sum_{q} q^{2} Q_{q} Q_{-q}=\frac{B_{2}}{2 A_{0}} \int d x\left(\partial_{x} Q\right)^{2}
\end{aligned}
$$

where $B_{2}=\sum_{l} A_{k l}(k-l)^{2}$, as defined in eq. (3). This immediately leads to the standard continuous version of the nonlinear $\sigma$-model:

$$
\mathcal{S}\{Q\}=-\frac{\pi \nu}{4} \operatorname{Str} \int d x\left[\frac{1}{2} D_{0}\left(\partial_{x} Q\right)^{2}+i \omega Q \Lambda\right]
$$

with the classical diffusion constant $D_{0}=\pi \nu B_{2}$, which implies the exponential localization of eigenstates with the localization length $\xi=\pi \nu D_{0} \propto b^{2}$.

\footnotetext{
${ }^{2}$ The expression for $A_{0}$ is valid for $\alpha>1 / 2$ and in the limit $N \rightarrow \infty$. When $\alpha<1 / 2, A_{0}$ starts to depend on $N$ which can be removed by a proper rescaling of the matrix elements $H_{i j}$ in eq. (1). Then, the properties of the model with $\alpha<1 / 2$ turn out to be equivalent to those of the GOE, so we will not consider this case any longer.
} 
Let us try to implement the same procedure for the present case of power-like bandshape, eq. (5). Restricting ourselves to the lowest order term in the momentum expansion, one arrives again at eq. (11) as long as $\alpha \geq 3 / 2$. This suggests that for $\alpha \geq 3 / 2$ the eigenstates of the present model should be localized in the spatial domain of the extension $\xi \propto \nu D_{0}$. However, in contrast to the usual RBM model we expect this localization to be power-like rather than exponential: $|\psi(r)|^{2} \propto r^{-2 \alpha}$ at $r \gg \xi$. This is quite evident due to the possibility of direct hopping with the same power-law. On a more formal level the appearance of powerlaw tails of wavefunctions is a consequence of the breakdown of the momentum expansion

for the function $A_{q}^{-1}-A_{0}^{-1}$ in higher orders in $q^{2}$. The presence of power-law "tails" of the wave functions, with an exponent $\alpha$ determined by the decay of hopping elements, was found in numerical simulations in Ref. [8].

The most interesting region $1 / 2<\alpha<3 / 2$ requires a separate consideration. The matter is that eq. (10) loses its validity in view of the divergency of the coefficient $B_{2}$. Instead, a close inspection shows that

$$
\begin{aligned}
A_{0}^{2}\left(A_{q}^{-1}-A_{0}^{-1}\right) & \approx A_{0}-A_{q}=2 \int_{0}^{\infty} d r a^{2}(r)(1-\cos q r) \\
& =\frac{2}{|q|}\left\{\int_{0}^{b|q|} d x(1-\cos x)+(b|q|)^{2 \alpha} \int_{b|q|}^{\infty} \frac{d x}{x^{2 \alpha}}(1-\cos x)\right\} \\
& \approx c_{\alpha} b^{2 \alpha}|q|^{2 \alpha-1} \text { for } 1 / 2<\alpha<3 / 2 \text { and }|q| \ll 1 / b,
\end{aligned}
$$

where $c_{\alpha}=2 \int_{0}^{\infty} \frac{d x}{x^{2 \alpha}}(1-\cos x)$ is a numerical constant.

The corresponding long wavelength part of the action,

$$
\mathcal{S}_{0}\{Q\}=-\frac{1}{t} \operatorname{Str} \int d q|q|^{2 \alpha-1} Q_{q} Q_{-q},
$$

can not be reduced to the local-in-space form in the coordinate representation any longer. Here, $1 / t=\frac{1}{4}(\pi \nu)^{2} c_{\alpha} b^{2 \alpha} \propto b^{2 \alpha-1} \gg 1$ plays the role of coupling constant, justifying the perturbative/renormalization group treatment of the model described in the next sections.

Let us mention, that considering the RBM model as a tight-binding Hamiltonian, the corresponding classical motion described by the master equation on the same $1 D$ lattice is superdiffusive for $1 / 2<\alpha<3 / 2:\langle|r|\rangle \propto t^{1 /(2 \alpha-1)}$. As will be discussed in Sec. VI, this influences the asymptotic behavior of the spectral correlation function for the corresponding quantum system.

\section{PERTURBATIVE TREATMENT OF THE NON-LINEAR $\sigma$-MODEL: GENERAL FORMULAS.}

In this section, we derive one-loop perturbative corrections to the density-density correlation function and inverse participation ratios. The analysis of these expressions for various values of the power-law parameter $\alpha$ will be presented in Sec. $\square$. 


\section{A. Density-density correlation function.}

The basic object characterizing the behavior of a particle in a random medium is the density-density correlation function, which can be generally expressed in terms of the $\sigma-$ model as follows [12]:

$$
K\left(r_{1}, r_{2} ; \omega\right)=-(\pi \nu)^{2} \int D Q Q_{12, \alpha \beta} k_{\beta \beta} Q_{21, \beta \alpha} e^{-S\{Q\}} .
$$

Here the indices $p, p^{\prime}$ of the matrix $Q_{p p^{\prime}, \alpha \beta}$ correspond to its advanced-retarded block structure, whereas $\alpha, \beta$ discriminate between bosonic and fermionic degrees of freedom. The matrix $k_{\beta \beta}$ is equal to 1 for bosons and (-1) for fermions. In order to calculate the correlation function (14) perturbatively, one needs to parametrize the matrix $Q$ in terms of the independent degrees of freedom. We find it convenient to use the following parametrization [12]:

$$
Q=\Lambda\left(W+\sqrt{1+W^{2}}\right)=\Lambda\left(1+W+\frac{W^{2}}{2}-\frac{W^{4}}{8}\right),
$$

where $W$ is block-off-diagonal in the advanced-retarded representation. To get the perturbative expansion for $K\left(r_{1}, r_{2} ; \omega\right)$, one has to substitute eq. (15) into (14), to separate the part quadratic in $W$ from the rest in the exponent and to apply the Wick theorem (see Ref. [14] for the contraction rules). In the usual case, when the action is given by eq. (11), the leading order (tree level) result reads in momentum space as follows:

$$
K_{0}(q, \omega)=\frac{2 \pi \nu}{D_{0} q^{2}-i \omega} .
$$

The perturbative quantum corrections do not modify the general form (16), but change the value of the diffusion constant. In particular, in one-loop order one gets eq. (16) with $D_{0}$ replaced by [15]

$$
D=D_{0}\left\{1-\frac{1}{\pi \nu V} \sum_{q_{i}=\pi n_{i} / L_{i}} \frac{1}{D_{0} q^{2}-i \omega}\right\} .
$$

This induces the standard weak-localization correction to the conductivity.

Now we implement an analogous procedure for the non-local $\sigma$-model of the type of eq. (13):

$$
\mathcal{S}\{Q\}=\frac{1}{t} \operatorname{Str} \sum_{r . r^{\prime}} U\left(r-r^{\prime}\right) Q(r) Q\left(r^{\prime}\right)-i \frac{\pi \nu}{4} \sum_{r} \operatorname{Str} \Lambda Q(r),
$$

with the Fourier-transform of $U(r)$ behaving at small momenta as

$$
\tilde{U}(q)=-|q|^{\sigma} ; \quad 1 / 2<\sigma<2 .
$$

The exponent $\sigma$ is related to the parameter $\alpha$ of the RBM model by $\sigma=2 \alpha-1$. In leading order, we keep in the action the terms quadratic in $W$ only, which yields: 


$$
K_{0}(q, \omega)=\frac{2 \pi \nu}{8(\pi \nu t)^{-1}|q|^{\sigma}-i \omega}
$$

corresponding to a superdiffusive behavior.

To calculate the one-loop correction to $K_{0}(q)$ (we set $\omega=0$ for simplicity) we expand the kinetic term in $\mathcal{S}\{Q\}$ up to fourth order in $W$ :

$$
\left.\sum_{r, r^{\prime}} \operatorname{Str} U\left(r-r^{\prime}\right) Q(r) Q\left(r^{\prime}\right)\right|_{4 \text { th order }}=\sum_{r, r^{\prime}} \frac{1}{4} \operatorname{Str} W^{2}(r) W^{2}\left(r^{\prime}\right) .
$$

The contraction rules are given by eqs. (8), (16) of Ref. [14, with the propagator $\Pi(q)$ replaced by

$$
\Pi(q)=\frac{t}{8|q|^{\sigma}}
$$

There is only one one-loop diagram contributing to the self-energy part in the present parametrization, see Fig. 1. Evaluating it, we find:

$$
\delta \Gamma_{1}^{(2)}=\frac{1}{2} \int(d k) \frac{|q+k|^{\sigma}}{|k|^{\sigma}}
$$

where $\int(d k) \equiv \int \frac{d k}{2 \pi} \equiv N \sum_{k}$. There is also a contribution to $\Gamma^{(2)}$ from the Jacobian of transformation (15), which is equal to 16

$$
\delta \Gamma_{2}^{(2)}=-\frac{1}{2} \delta(0) \equiv-\frac{1}{2} \int(d k)
$$

Combining eqs. (23) and (24), we get

$$
\delta \Gamma^{(2)}=\frac{1}{2} \int(d k) \frac{|q+k|^{\sigma}-|k|^{\sigma}}{|k|^{\sigma}} .
$$

Finally, we get the following expression for the density-density correlation function up to one-loop order:

$$
K^{-1}(q)=K_{0}^{-1}(q)-\frac{(\pi \nu)^{2}}{2} \int(d k) \frac{|q+k|^{\sigma}-|k|^{\sigma}}{|k|^{\sigma}} .
$$

\section{B. Inverse participation ratio.}

In order to characterize eigenfunctions statistics quantitatively, it is convenient to introduce a set of moments $I_{q}=\sum|\psi(r)|^{2 q}$ of the eigenfunction local intensity $|\psi(r)|^{2}$. The quantity $I_{2}$ is known as the inverse participation ratio (IPR). The whole set of moments $I_{q}$ is a useful measure of the eigenfunction structure. For completely "ergodic" eigenfunctions covering randomly but uniformly the whole sample, $\left\langle I_{q}\right\rangle=\frac{(2 q-1) ! !}{N^{q-1}}$, as in GOE. In contrast, if eigenfunctions are localized in a domain of size $\xi$, then $\left\langle I_{q}\right\rangle \propto 1 / \xi^{q-1}$. Finally, the multifractal structure of the wavefunction manifests itself via the dependence $\left\langle I_{q}\right\rangle \propto 1 / N^{d_{q}(q-1)}$ with $d_{q}<1$ being the set of fractal dimensions 17, 18. 
The method allowing one to calculate perturbative corrections to the GOE-like results in the weak localization regime was developed in [19,14]. It is straightforwardly applicable to the present case of power-law RBM, provided the appropriate modification of the diffusion propagator entering the contraction rules is made, see the text preceding eq. (22). One finds

$$
\left\langle I_{q}\right\rangle=\left\{1+\frac{1}{N} q(q-1) \sum_{r} \Pi(r, r)\right\} \frac{(2 q-1) ! !}{N^{q-1}},
$$

where $\Pi\left(r, r^{\prime}\right)=(1 / N) \sum_{q} \Pi(q) \exp \left[i q\left(r-r^{\prime}\right)\right]$ and $\Pi(q)$ is given by eq. (22).

\section{RENORMALIZATION GROUP TREATMENT.}

Our effective $\sigma$-model, eq. (18), is actually of one-dimensional nature. However, for the sake of generality, in the present section we find it convenient to consider it to be defined in $d$-dimensional space with arbitrary $d$. The form (20) of the generalized diffusion propagator suggests that $d=\sigma$ should play the role of the logarithmic dimension for the problem. In the vicinity of this critical value it is natural to carry out a renormalization group $(\mathrm{RG})$ treatment of the model. We will follow the procedure developed for general non-linear $\sigma$-models in [16]. We start from expressing the action in terms of the renormalized coupling constant $t=Z_{1}^{-1} t_{B} \mu^{d-\sigma}$, where $t_{B}$ is the bare coupling constant and $\mu^{-1}$ is the length scale governing the renormalization $[$ :

$$
\begin{aligned}
S & =\frac{\mu^{d-\sigma}}{2 t Z_{1}} \sum_{r r^{\prime}} U\left(\left|r-r^{\prime}\right|\right) \operatorname{Str}\left[-W(r) W\left(r^{\prime}\right)+\sqrt{1+W^{2}(r)} \sqrt{1+W^{2}\left(r^{\prime}\right)}\right] \\
& -\frac{i \pi \nu \omega}{4} \sum_{r} \operatorname{Str} \sqrt{1+W^{2}(r)} .
\end{aligned}
$$

Expanding the action in powers of $W(r)$ and keeping terms up to 4-th order, we get

$$
\begin{aligned}
S & =S_{0}+S_{1}+O\left(W^{6}\right), \\
S_{0} & =\frac{\mu^{d-\sigma}}{4 t Z_{1}} \sum_{r r^{\prime}} U\left(\left|r-r^{\prime}\right|\right) \operatorname{Str}\left(W(r)-W\left(r^{\prime}\right)\right)^{2}-\frac{i \pi \nu \omega}{8} \sum_{r} \operatorname{Str} W^{2}(r), \\
S_{1} & =\frac{\mu^{d-\sigma}}{8 t Z_{1}} \sum_{r r^{\prime}} U\left(\left|r-r^{\prime}\right|\right) \operatorname{Str} W^{2}(r) W^{2}\left(r^{\prime}\right)+\frac{i \pi \nu \omega}{32} \sum_{r} \operatorname{Str} W^{4}(r) .
\end{aligned}
$$

We have restricted ourselves to 4-th order terms, since they are sufficient for obtaining the renormalized quadratic part of the action in one-loop order. The calculation yields, after the cancellation of an $\int(d k) \propto \delta(0)$ term with the contribution of the Jacobian:

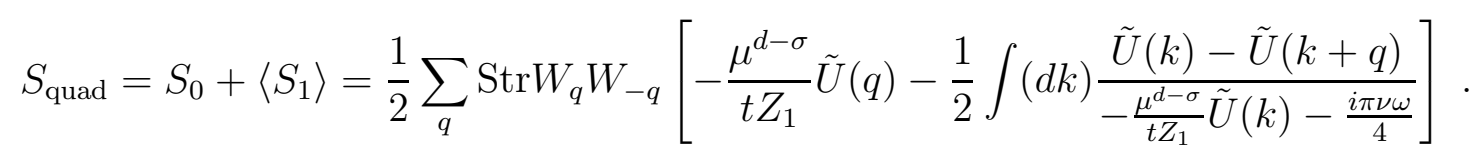

\footnotetext{
${ }^{3}$ Note that the $W$-field renormalization is absent due to the supersymmetric character of the problem, which is physically related to the particle number conservation [12].
} 
According to the renormalization group idea, one has to chose the constant $Z_{1}(t)=1+a t+\ldots$ so as to cancel the divergency in the coefficient in front of the leading $|q|^{\sigma}$ term. This will be done in the next section.

\section{ANALYSIS OF THE MODEL FOR DIFFERENT VALUES OF THE EXPONENT $\sigma=2 \alpha-1$.}
A. $1<\sigma<2(1<\alpha<3 / 2)$.

To evaluate the one-loop correction (26) to the diffusion propagator, we use the expansion

$$
\frac{|\boldsymbol{q}+\boldsymbol{k}|^{\sigma}}{|\boldsymbol{k}|^{\sigma}}-1 \simeq\left\{\begin{array}{ll}
\sigma \frac{\boldsymbol{q} \boldsymbol{k}}{k^{2}}+\frac{\sigma}{2} \frac{q^{2}}{k^{2}}+\sigma\left(\frac{\sigma}{2}-1\right)\left(\frac{\boldsymbol{q} \boldsymbol{k}}{k^{2}}\right)^{2}+\ldots, & q \ll k \\
\frac{|q|^{\sigma}}{|k|^{\sigma}}, & q \gg k
\end{array} .\right.
$$

Thus, the integral in eq. (26) can be estimated as

$$
I \equiv \int(d k)\left[\frac{|\boldsymbol{q}+\boldsymbol{k}|^{\sigma}}{|\boldsymbol{k}|^{\sigma}}-1\right] \approx \int_{k>q}(d k) \sigma\left(1+\frac{\sigma-2}{d}\right) \frac{q^{2}}{2 k^{2}}+\int_{k<q}(d k) \frac{|q|^{\sigma}}{|k|^{\sigma}} .
$$

For $\sigma>d=1$ the integral diverges at low $k$, and the second term in eq. (32) dominates. This gives

$$
I \sim L^{\sigma-1}|q|^{\sigma}
$$

where $L$ is the system size determining the infrared cut-off (in the original RBM formulation

it is just the matrix size $N$ ). This leads to the following one-loop expression for the diffusion correlator

$$
K(q)=\frac{(\pi \nu)^{2} \tilde{t}}{4|q|^{\sigma}} ; \quad \tilde{t}^{-1}=t^{-1}-\operatorname{const} L^{\sigma-1}
$$

Now we turn to the renormalization group analysis, as described in Sec. IV]. In oneloop order, the expression for the renormalization constant $Z_{1}$ is determined essentially by the same integral $I$, eq. (32), with the RG scale $\mu$ playing the role of the infrared cut-off (analogous to that of the system size $L$ in eq. (34)). This yields (in the minimal subtraction scheme):

$$
Z_{1}(t)=1-\frac{1}{2 \pi} \frac{t}{\sigma-1}+O\left(t^{2}\right)
$$

It is easy to check that this results in a relation between the bare and the renormalized coupling constant analogous to eq. (34):

$$
\frac{1}{t} \mu^{1-\sigma} \equiv \frac{Z_{1}}{t_{B}}=\frac{1}{t_{B}}-\frac{1}{2} \frac{1}{\sigma-1} \mu^{1-\sigma} .
$$


From eq. (35), we get the expression for the $\beta$-function:

$$
\beta(t)=\left.\frac{\partial t}{\partial \ln \mu}\right|_{t_{B}}=\frac{(1-\sigma) t}{1+t \partial_{t} \ln Z_{1}(t)}=-(\sigma-1) t-\frac{t^{2}}{2 \pi}+O\left(t^{3}\right) .
$$

Both eqs. (34) and (37) show that the coupling constant $t$ increases with the system size $L$ (resp. scale $\mu$ ), which is analogous to the behavior found in the conventional scaling theory of localization in $d<2$ dimensions [20,21]. The $\mathrm{RG}$ flow reaches the strong coupling regime $t \sim 1$ at the scale $\mu \sim t_{B}^{1 /(\sigma-1)}$. Remembering the relation of the bare coupling constant $t_{B}$ and the index $\sigma$ to the parameters of the original PRBM model: $t_{B}^{-1} \propto b^{2 \alpha-1} ; \sigma \propto 2 \alpha-1$, we conclude that the length scale

$$
\xi \sim t_{B}^{-1 /(\sigma-1)} \sim b^{\frac{2 \alpha-1}{2 \alpha-2}}
$$

plays the role of the localization length for the PRBM model.

This conclusion is also supported by an inspection of the expression for the IPR, eq. (27). Evaluating the one-loop perturbative correction in eq. (27), one gets

$$
\left\langle I_{q}\right\rangle=\frac{(2 q-1) ! !}{N^{q-1}}\left\{1+q(q-1) \frac{t}{8 \pi^{\sigma}} \zeta(\sigma) N^{\sigma-1}\right\}
$$

where $\zeta(\sigma)$ is Riemann's zeta-function; $\zeta(\sigma) \simeq 1 /(\sigma-1)$ for $\sigma$ close to unity. The correction term becomes comparable to the leading (GOE) contribution for the system size $N \sim t^{1 /(\sigma-1)}$, parametrically coinciding with the localization length $\xi$. For larger $N$ the perturbative expression (39) loses its validity, and the IPR is expected to saturate at a constant value $\left\langle I_{q}\right\rangle \sim \xi^{1-q}$ for $N \gg \xi$.

In conclusion of this subsection, let us stress once more that the localized eigenstates in the present model are expected to have integrable power-law tails: $\left|\psi^{2}(r)\right| \propto r^{-2 \alpha}=r^{-\sigma-1}$ at $r \gg \xi$.

$$
\text { B. } 0<\sigma<1 \quad(1 / 2<\alpha<1)
$$

We start again from considering the perturbative corrections to the diffusion propagator (26). The integral (32) is now dominated by the region $k \sim q$, and is proportional to $|q|$. We get, therefore:

$$
(\pi \nu)^{2} K^{-1}(q)=\frac{4|q|^{\sigma}}{t}-C_{\sigma}|q|
$$

with a numerical constant $C_{\sigma}$.

We see that the correction term is of higher order in $|q|$ as compared to the leading one. Thus, it does not lead to a renormalization of the coupling constant $t$. This is readily seen also in the framework of the RG scheme, where the one-loop integral in eq. (30) gives no rise to terms of the form $|q|^{\sigma}$. One can check that this feature is not specific to the one-loop RG calculation, but holds in higher orders as well. An analogous conclusion was reached for the case of a vector model with long-ranged interaction by Brezin et al. [22]. 
In our case this implies that the renormalization constant $Z_{1}$ is equal to unity and the $\beta$-function is trivial:

$$
\beta(t)=(1-\sigma) t
$$

This means that the model does not possess a critical point, and, for $\sigma<1$, all states are delocalized, for any value of the bare coupling constant $t$. This property should be contrasted with the behavior of a $d$-dimensional conductor described by the conventional local non-linear $\sigma-$ model and undergoing an Anderson transition at some critical coupling $t=t_{c}[21]$.

Since all states of the model turn out to be delocalized, one might think that their statistical properties are the same as for GOE. This is, however, not quite true. In particular, calculating the variance of the inverse participation ratio $I_{2}$ in the same way as it is done in Ref. [14] we get:

$$
\delta(I)=\frac{\left\langle I_{2}^{2}\right\rangle-\left\langle I_{2}\right\rangle^{2}}{\left\langle I_{2}\right\rangle^{2}}=\frac{8}{N^{2}} \sum_{r r^{\prime}} \Pi^{2}\left(r, r^{\prime}\right)=\frac{8}{N^{2}} \sum_{q=\pi n / N ; n=1,2, . .} \Pi^{2}(q) .
$$

At $\sigma>1 / 2$ the sum over $q$ is convergent yielding

$$
\delta(I)=\frac{t^{2}}{8 \pi^{2 \sigma}} \frac{\zeta(2 \sigma)}{N^{2-2 \sigma}} .
$$

Thus, in this regime the fluctuations of the IPR are much stronger than for the GOE where $\delta(I) \propto 1 / N$. Only for $\sigma<1 / 2(\alpha<3 / 4)$ the IPR fluctuations acquire the GOE character.

Considering higher cumulants of the IPR, $\left\langle\left\langle I_{2}^{n}\right\rangle\right\rangle$, one finds that the GOE behavior is restored at $\sigma<\sigma_{c}^{(n)} \equiv 1 / n$. In this sense, the model is analogous to a $d$-dimensional conductor at $d=2 / \sigma$. Therefore, only when $\sigma \rightarrow 0$ (correspondingly, $\alpha \rightarrow 1 / 2$ in the original PRBM formulation) all statistical properties become equivalent to those typical for the GOE.

\section{Critical regime: $\sigma=1(\alpha=1)$.}

As we have seen, the case $\sigma=1$ separates the regions of localized $(\sigma>1)$ and extended $(\sigma<1)$ states. It is then natural to expect some critical properties showing up just at $\sigma=1$. Let us again start from considering the generalized diffusion propagator, eq. (26). At $\sigma=1$ the one-loop correction yields

$$
\frac{1}{(\pi \nu)^{2}} K^{-1}(q)=4|q|\left[t^{-1}-\frac{1}{2} \ln (|q| L)\right] .
$$

As it was natural to expect for the critical point, the correction to the coupling constant is of logarithmic nature. However, eq. (44) differs essentially from that typical for a $2 D$ disordered conductor:

$$
t^{-1}=t_{B}^{-1}-\ln (L / l)
$$


where the bare coupling constant $t_{B}$ corresponds to scale $l$. Comparing the two formulas, we see that in eq. (44) the mean free path $l$ is replaced by the inverse momentum $q^{-1}$. Therefore, the correction to the bare coupling constant is small for low momenta $q \sim 1 / L$, and the correlator $K(q)$ is not renormalized. This implies the absence of eigenstate localization, in contrast to the $2 D$ diffusive conductor case, where eq. (45) results in an exponentially large localization length $\xi \propto \exp t_{B}^{-1}$. On a more formal level, the absence of essential corrections to the low- $q$ behavior of $K(q)$ is due to the fact that the region $k>q$ does not give a logarithmic contribution. This is intimately connected with the absence of $t$ renormalization at $\sigma<1$.

To study in more details the structure of critical eigenfunctions, let us consider the set of IPR, $I_{q}$. The perturbative correction, eq. (27), is evaluated at $\sigma=1$ as

$$
\left\langle I_{q}\right\rangle=\left\{1+q(q-1) \frac{t}{8 \pi} \ln (N / b)\right\} \frac{(2 q-1) ! !}{N^{q-1}},
$$

where the microscopic scale $b$, eq. (5), enters as the ultraviolet cut-off for the $\sigma$-model, the role usually played by the mean free path $l$. This formula is valid as long as the correction is small: $q \ll\left[\frac{t}{8 \pi} \ln (N / b)\right]^{-1 / 2}$. For larger $q$ the perturbation theory breaks down, and one has to use the renormalization group approach. This procedure, pioneered by Wegner [17] and developed by Altshuler, Kravtsov and Lerner [23] requires the introduction of higher vertices of the type $z_{q} \int \operatorname{Str}^{q}(Q k \Lambda) d r$ in the action of the non-linear $\sigma$-model, and their subsequent renormalization. This results in RG equations for the charges $z_{q}$ which in the present case, and in one-loop order, read:

$$
\frac{d z_{q}}{d \ln \mu^{-1}}=q(q-1) \frac{t}{8 \pi} z_{q}
$$

where $\mu^{-1}$ is the renormalization scale. Integrating eq. (47), we find:

$$
\left\langle I_{q}\right\rangle=\frac{(2 q-1) ! !}{N^{q-1}}\left(\frac{N}{b}\right)^{q(q-1) \frac{t}{8 \pi}} .
$$

Note, that this formula is reduced to the perturbative expression, eq. (46), in the regime $q \ll\left[\frac{t}{8 \pi} \ln (N / b)\right]^{-1 / 2}$.

The behavior described by eq. (48) is characteristic for a multifractal structure of wave functions, when

$$
\left\langle I_{q}\right\rangle \propto N^{-d_{q}(q-1)}
$$

with $d_{q}$ being the set of fractal dimensions. We find from eq. (48):

$$
d_{q}=1-q \frac{t}{8 \pi} .
$$

This form of the fractal dimensions is similar to that found in two and $2+\epsilon$ dimensions for the usual diffusive conductor [17, [18,23]. The one-loop result (50) holds for $q \ll 8 \pi / t$.

The set of fractal dimensions $d_{q}$ (as well as spectral properties at $\sigma=1$, see next section) is parametrized by the coupling constant $t$. Strictly speaking, our $\sigma$-model derivation is 
justified for $t \ll 1$ (i. e. $b \gg 1$ ). However, the opposite limiting case can be also studied, following the ideas of Levitov [9]. This corresponds to a $d$-dimensional Anderson insulator, perturbed by a weak long-range hopping with an amplitude decreasing with distance as $r^{-\sigma}$. The arguments of Levitov [9] suggest that the states delocalize at $\sigma \leq d$, carrying some fractal properties at $\sigma=d$. Our PRBM model in the limit $b \ll 1$ is just the $1 D$ version of this problem. This shows that the conclusion about localization (delocalization) of eigenstates for $\sigma>1$ (resp. $\sigma<1$ ), with $\sigma=1$ being a critical point holds irrespective of the particular value of the parameter $b$. Alternatively, the regime of the Anderson insulator with weak power-law hopping can be described in the framework of the non-linear $\sigma$-model, eq. (18), by considering the limit $t \gg 1$. Formally, the non-linear $\sigma$-model for arbitrary $t$ can be derived from a microscopic tight-binding model by allowing $n \gg 1$ "orbitals" per site [21].

At any rate, the PRBM model, eqs. (1), (5), with arbitrary $0<b<\infty$, or the $\sigma$-model, eq. (18), with arbitrary coupling constant $0<t<\infty$ display at $\sigma=1$ a rich critical behavior parametrized by the value of $b$ (respectively, $t$ ).

\section{SPECTRAL PROPERTIES.}

Let us consider now the issue of spectral statistics of the PRBM model. As is well known, a usual diffusive conductor exhibits the Wigner-Dyson (RMT) level statistics in the limit of infinite dimensionless conductance $g=2 \pi \nu D L^{d-2}$. At finite $g \gg 1$, there appear deviations [24, 19, 25]. In the present section we would like to address the analogous problem in the case of the PRBM model.

The basic quantity characterizing the spectral properties is the two-level correlation function

$$
R(s)=\frac{1}{\langle\nu\rangle^{2}}\langle\nu(E) \nu(E+\omega)\rangle,
$$

where $s=\omega / \Delta, \Delta$ is the mean level spacing, $\nu(E)$ is the density of states at energy $E$, and $\langle\ldots\rangle$ denotes the ensemble averaging. Following [19], we find the leading correction to the Wigner-Dyson form $R^{W D}(s)$ of the level correlation function, eq. (51), as

$$
R(s)=\left[1+\frac{1}{2} \mathcal{C} \frac{d^{2}}{d s^{2}} s^{2}\right] R^{W D}(s),
$$

where

$$
\mathcal{C}=\frac{1}{N^{2}} \sum_{r r^{\prime}} \Pi^{2}\left(r, r^{\prime}\right)=\frac{1}{N^{2}} \sum_{q=\pi n / N ; n=1,2, \ldots} \Pi^{2}(q)= \begin{cases}\frac{t^{2}}{64 \pi^{2 \sigma}} N^{2 \sigma-2}, & \sigma>1 / 2 \\ \operatorname{const} \frac{t^{2}}{64 \pi^{2 \sigma} b^{1-2 \sigma}} N^{-1}, & \sigma<1 / 2 .\end{cases}
$$

At $\sigma<1 / 2$ the sum divergent at high momenta is cut off at $q \sim \pi / b$; the procedure leaving undetermined a constant of order of unity. 
The correlation function $R(s)$ is close to its RMT value if $\sigma<1$ (the region of delocalized states), or else if $\sigma>1$ and the system size $N$ is much less than the localization length $\xi$, eq. (38). Under these conditions, eq. (52) holds as long as the correction term is small compared to the leading one. This requirement produces the following restriction on the frequency $s=\omega / \Delta$ :

$$
s<s_{c} \sim\left\{\begin{array}{ll}
t^{-1} N^{1-\sigma}, & \sigma>1 / 2 \\
t^{-1} b^{\frac{1}{2}-\sigma} N^{1 / 2} \propto(N b)^{1 / 2}, & \sigma<1 / 2
\end{array} .\right.
$$

At larger frequencies $\left(s>s_{c}\right)$, the form of the level correlation function changes from the $1 / s^{2}$ behavior typical for RMT to a completely different one [24]. Extending the calculation of Ref. [24] to the present case, we find

$$
\begin{aligned}
R(s) & =\frac{\Delta^{2}}{\pi^{2}} \operatorname{Re} \sum_{n=0,1, \ldots} \frac{1}{\left[\frac{8}{\pi \nu t}\left(\frac{\pi n}{N}\right)^{\sigma}-i \omega\right]^{2}} \\
& \propto\left\{\begin{array}{ll}
N^{1-1 / \sigma} t^{1 / \sigma} s^{1 / \sigma-2}, & \sigma>1 / 2 \quad(\sigma \neq 1) \\
t^{2} N^{-1} b^{2 \sigma-1} \propto(N b)^{-1}, & \sigma<1 / 2
\end{array} .\right.
\end{aligned}
$$

At last, let us consider the level statistics in the critical regime $\sigma=1$. In this case the coefficient of proportionality in the asymptotic expression (55) vanishes in view of analyticity:

$$
R(s) \sim \frac{\Delta t}{16 \pi^{2}} \int_{-\infty}^{\infty} \frac{d x}{(x-i \omega)^{2}}=0 .
$$

This is similar to what is known to happen in the case of a $2 D$ diffusive conductor [26,27]. A more accurate consideration requires taking into account the high-momentum cut-off at $q \sim b^{-1}$. In full analogy with the $2 D$ situation mentioned [26,28,27, we find then a linear term in the level number variance:

$$
\begin{aligned}
& \left\langle\delta N^{2}(E)\right\rangle \simeq \kappa\langle N(E)\rangle \\
& \kappa=\int R(s) d s=\frac{t}{8 \pi} .
\end{aligned}
$$

The presence of the linear term (57) (as well as the multifractality of eigenfunctions, Sec. V) makes the case $\sigma=1$ similar to the situation on the mobility edge of a disordered conductor in $d>2$ [28]. Let us finally mention that the value of $\kappa$, eq. (57), is in agreement with the formula $\kappa=\left(d-d_{2}\right) / 2 d$, suggested recently by Kravtsov [29], where $d_{2}$ is given by eq. (50]) with $q=2$, and $d=1$ in the present case.

\section{NUMERICAL SIMULATIONS}

We have performed numerical simulations of the PRBM model for values of $\alpha \in[0,2]$ and $b=1$. In Fig. 2 we present typical eigenfunctions for four different regions of $\alpha$. In agreement with the theoretical picture presented above, the eigenstates corresponding to

$\alpha=0.375$ and $\alpha=0.875$ are extended, whereas those corresponding to $\alpha=1.25$ and $\alpha=1.625$ are localized. At the same time, one can notice that the states with $\alpha=0.875$ 
and $\alpha=1.25$ exhibit a quite sparse structure, as opposed to the other two cases. We believe that this can be explained by the proximity of the former two values of $\alpha$ to the critical value $\alpha=1.0$, where eigenstates should show the multifractal behavior, see Sec. $\nabla$.

In order to get a more quantitative insight into the properties of the eigenstates, we concentrated our attention on the behavior of the mean value of the IPR, $\left\langle I_{2}\right\rangle$, and on the relative variance, $\delta=\left(\left\langle I_{2}^{2}\right\rangle-\left\langle I_{2}\right\rangle^{2}\right) /\left\langle I_{2}\right\rangle^{2}$. At any given $\alpha$ we studied the dependence of the quantities $\left\langle I_{2}\right\rangle$ and $\delta$ on the matrix size $N$ and approximated these dependencies by the power-laws $\left\langle I_{2}\right\rangle \propto 1 / N^{\nu}, \delta \propto 1 / N^{\mu}$ for $N$ ranging from 100 to 2400. On Figs. 3 and 4 we plotted the values of the exponents $\nu$ and $\mu$ obtained in this way, versus the PRBM parameter $\alpha$. The expected theoretical curves following from the results of Sec. $\square$ are presented as well. We see from Fig. 3 that the data show a crossover from the behavior typical for extended states $(\nu=1)$ to that typical for localized states $(\nu=0)$, centered approximately at the critical point $\alpha=1$. We attribute the deviations from the sharp steplike theoretical curve $\nu(\alpha)$ to the finite-size effects which are unusually pronounced in the PRBM model due to the long-range nature of the off-diagonal coupling. The data for the exponent $\mu$ (Fig. 4) also show a reasonable agreement with the expected linear crossover, $\mu=4(1-\alpha)$ for $3 / 4<\alpha<1$, see eq. (43).

\section{CONCLUSION.}

In this paper, we have performed a detailed investigation of the RBM model with a power-law decay of the matrix elements, eq. (5). Physically, one can look at this model as describing a particle in a $1 D$ disordered system with a power-law hopping term. As a theoretical tool, we have used a mapping of the problem onto a supermatrix non-linear $\sigma$-model, eqs. (18), (19). Depending on the value of the power-law exponent $\alpha$ in eq. (5) (or, equivalently, $\sigma=2 \alpha-1$ in eq. (19)), three different regimes are found: For $\alpha>1(\sigma>1)$ all eigenstates are localized with integrable power-law tails. For $\alpha<1$ $(\sigma<1)$ the eigenstates are delocalized, for any value of the bandwidth $b$ of the PRBM model, eq. (5) (resp. coupling constant $t \propto b^{1-2 \alpha}$ of the $\sigma$-model, eq. (19)). These two regimes are separated by the critical value $\alpha=1(\sigma=1)$, where the structure of eigenstates is multifractal, and energy levels show statistics intermediate between Wigner-Dyson and Poisson ones. These critical properties are similar to those found on the mobility edge of a $d$-dimensional disordered conductor. At $\sigma=1$, we find a family of critical points labeled by the value of the coupling constant $t$ of the non-linear $\sigma$-model, so that the critical behavior is parametrized by the value of $t$. In particular, it determines the multifractal exponents $d_{q}$ and the coefficient $\kappa$ of the linear term in the level number variance, which are given at $t \ll 1$ by eqs. (50) and (57), respectively.

Turning our attention to the regime of localized states, we find that it can be subdivided into two domains. In the region $\alpha>3 / 2$ the properties of the model are rather close to those of a conventional quasi-1D conductor [3]. On the other hand, for $1<\alpha<3 / 2$ the wave packet spreading on a short time scale is superdiffusive, $\langle|r|\rangle \sim t^{1 /(2 \alpha-1)}$, which leads to a modification of the Altshuler-Shklovskii "tail" of the spectral correlation function, eq. (55), and to an unusual scaling of the localization length $\xi \propto b^{2 \alpha-1}$. The regime of extended states, $\alpha<1$, can be also subdivided into two domains. For $\alpha<1 / 2$, all statistical properties are identical to those of the GOE (which corresponds to $\alpha=0$ ). On the other 
hand, at $1 / 2<\alpha<1$ the model is quite similar to a diffusive conductor in $d=(\alpha-1 / 2)^{-1}$ dimensions. This is reflected, in particular, in the fluctuations of the IPR (see eq. (43) and the discussion following it), and in the large-frequency "tail" of the spectral correlator, eq. (55).

Our conclusion about the existence of a set of critical theories at $\alpha=1$, parametrized by the coupling constant $t$, perfectly agrees with earlier results by Levitov [9]. He studied the effect of a weak power-law hopping on an Anderson insulator, which corresponds to the limit $t \gg 1$ in the $\sigma$-model formulation, and arrived at the conclusion of criticality of the model at $\alpha$ equal to the spatial dimension $d$. Let us also note that our results are in accordance with the fact of absence of localization effects in the Quantum Fermi Accelerator model [7]. As it was pointed out in []], the evolution equation for this model takes the form of a finite difference equation of the tight binding type with a long range hopping term decaying in a power-law fashion, eq. (5), with $\alpha=1$. Our results show that this case corresponds to the critical point with extended eigenstates and intermediate level statistics, in agreement with the behavior found in Ref. [7].

We have presented results of a direct numerical simulation of the PRBM model. Our data are in reasonable agreement with the above theoretical picture. However, a more detailed numerical investigation of the structure of eigenstates and of spectral statistics is certainly desirable. In particular, it would be very interesting to study the critical manifold, $\alpha=1$, where the multifractal properties of eigenstates and intermediate level statistics are predicted by our theory.

\section{ACKNOWLEDGMENTS.}

We are grateful to F. Izrailev and I. Guarneri for useful discussions on early stage of this work, and to V. E. Kravtsov, M. R. Zirnbauer, B. L. Altshuler, and A. M. Tsvelik for discussion of the results. A.D.M. and Y.V.F. acknowledge with thanks the warm hospitality extended to them during the Program "Quantum Chaos in Mesoscopic Systems" in the Institute for Theoretical Physics in Santa Barbara, where this research was completed. This research was supported in part by the National Science Foundation under Grant No. PHY9407194 (A.D.M. and Y.V.F.), the Deutsche Forschungsgemeinschaft by SFB 195 (A.D.M.) and SFB 237 "Unordnung und Große Fluktuationen" (Y.V.F.), the Minerva Foundation (F.-M.D.), and the Minerva Center for Nonlinear Physics of Complex Systems (Y.V.F. and F.-M.D.). 


\section{REFERENCES}

$\dagger$ On leave from Petersburg Nuclear Physics Institute, 188350 Gatchina, St.Petersburg, Russia.

[1] T. H. Seligman, J. J. M. Verbaarschot, and M. R. Zirnbauer, J. Phys. A: Math. Gen. 18, $2751(1985)$.

[2] V. V. Flambaum, A. A. Gribakina, G. F. Gribakin, and M. G. Kozlov, Phys. Rev. A 50, 267 (1994).

[3] Y. V. Fyodorov, A. D. Mirlin, Int. J. Mod. Phys. B 8, 3795 (1994).

[4] F. M. Izrailev, Chaos, Solitons, Fractals, 5, 1219 (1995); G. Casati, L. Molinari, and F. M. Izrailev, Phys. Rev. Lett. 67, 2405 (1991).

[5] F. M. Izrailev, Phys. Rep. 196, 299 (1990).

[6] S. Fishman, D. R. Grempel, R. E. Prange, Phys. Rev. Lett. 49, 509 (1982).

[7] J. V. Jose and R. Cordery, Phys. Rev. Lett. 56, 290 (1986).

[8] G. Yeung and Y. Oono, Europhys. Lett. 4, 1061 (1987).

[9] L. Levitov, Europhys. Lett. 9, 83 (1989); Phys. Rev. Lett. 64, 547 (1990).

[10] A. V. Balatsky and M. I. Salkola, Phys. Rev. Lett. 76, 2386 (1996); B. L. Altshuler and L. Levitov, private communication.

[11] Y. V. Fyodorov and A. D. Mirlin, Phys. Rev. Lett. 67, 2405 (1991); Phys. Rev. Lett. 69, 1093 (1992); Phys. Rev. Lett. 71, 412 (1993); A. D. Mirlin and Y. V. Fyodorov, J. Phys. A: Math. Gen. 26, L551 (1993).

[12] K. B. Efetov, Adv. Phys. 32, 53 (1983).

[13] J. J. M. Verbaarschot, H. A. Weidenmüller, and M. R. Zirnbauer, Phys. Rep. 129, 367 (1985).

[14] Y. V. Fyodorov and A. D. Mirlin, Phys. Rev. B 51, 13403 (1995).

[15] L. P. Gor'kov, A. I. Larkin, and D. E. Khmelnitskii, JETP-Lett. 30, 228 (1979).

[16] E. Brézin and J. Zinn-Justin, Phys. Rev. B 14, 3110 (1976); E. Brézin, J. Zinn-Justin, and J. C. Le Guillou, Phys. Rev. D 14, 2615 (1976); E. Brézin, S. Hikami, and J. Zinn-Justin, Nucl. Phys. B 165, 528 (1980); J. Zinn-Justin, Quantum Field Theory and Critical Phenomena, Clarendon Press, Oxford, 1989.

[17] F. Wegner, Z. Phys. B 36, 209 (1980).

[18] C. Castellani and L. Peliti, J. Phys. A: Math. Gen. 19, L429 (1986).

[19] V. E. Kravtsov and A. D. Mirlin, Pis'ma Zh. Eksp. Theor. Fiz. 60, 645 (1994) [JETP Lett. 60, 656 (1994)].

[20] E. Abrahams, P. W. Anderson, D. C. Licciardello, and T. V. Ramakrishnan, Phys. Rev. Lett. 42, 673 (1979).

[21] F. Wegner, Phys. Rep. 67, 15 (1980).

[22] E. Brézin, J. Zinn-Justin, and J. C. Le Guillou, J. Phys. A: Math. Gen. 9, L119 (1976).

[23] B. L. Altshuler, V. E. Kravtsov, I. V. Lerner, in Mesoscopic Phenomena in Solids, ed. by B. L. Altshuler et al. (North Holland, Amsterdam, 1991).

[24] B. L. Altshuler and B. I. Shklovski, Zh. Eksp. Teor. Fiz. 91, 220 (1986) [Sov. Phys. JETP 64, 127 (1986)].

[25] A. V. Andreev and B. L. Altshuler, Phys. Rev. Lett. 75, 902 (1995).

[26] A. Altland and Y. Gefen, Phys. Rev. Lett. 71, 3339 (1993); Phys. Rev. B 51, 10671 (1995).

[27] V. E. Kravtsov and I. V. Lerner, Phys. Rev. Lett. 74, 2563 (1995). 
[28] A. G. Aronov and A. D. Mirlin, Phys. Rev. B 51, 6131 (1995).

[29] V. E. Kravtsov, unpublished. 


\section{Figure captions}

Fig. 1

One-loop diagram contributing to the self-energy part of the density-density correlation function. The solid line denotes the $Q$-field propagator, whereas the dashed line represents the interaction $U\left(r-r^{\prime}\right)$.

\section{Fig. 2}

Typical eigenfunctions for the matrix size $N=800$ and four different values of $\alpha$ : a) $\alpha=0.375$; b) $\alpha=0.875$; c) $\alpha=1.250$; d) $\alpha=1.625$.

\section{Fig. 3}

Index $\nu$ characterizing the dependence of the inverse participation ratio $\left\langle I_{2}\right\rangle$ on the matrix size $N$ via $\left\langle I_{2}\right\rangle \propto 1 / N^{\nu}$, as a function of $\alpha$. Points refer to the best-fit values obtained from matrix sizes between $N=100$ and $N=1000$ (squares) or $N=2400$ (circles). The dashed line is the theoretical prediction for the transition from $\nu=1$, at small $\alpha$, to $\nu=0$, at large $\alpha$.

\section{Fig. 4}

The same as Fig. 3, but for the index $\mu$, derived from the $N$ dependence of the variance $\delta$ of the inverse participation ratio: $\delta \equiv\left(\left\langle I_{2}^{2}\right\rangle-\left\langle I_{2}\right\rangle^{2}\right) /\left\langle I_{2}\right\rangle^{2} \propto 1 / N^{\mu}$. The dashed line corresponds to the predicted linear crossover from $\mu=1$ at $\alpha<3 / 4$ to $\mu=0$ at $\alpha>1$. 


$$
\zeta
$$




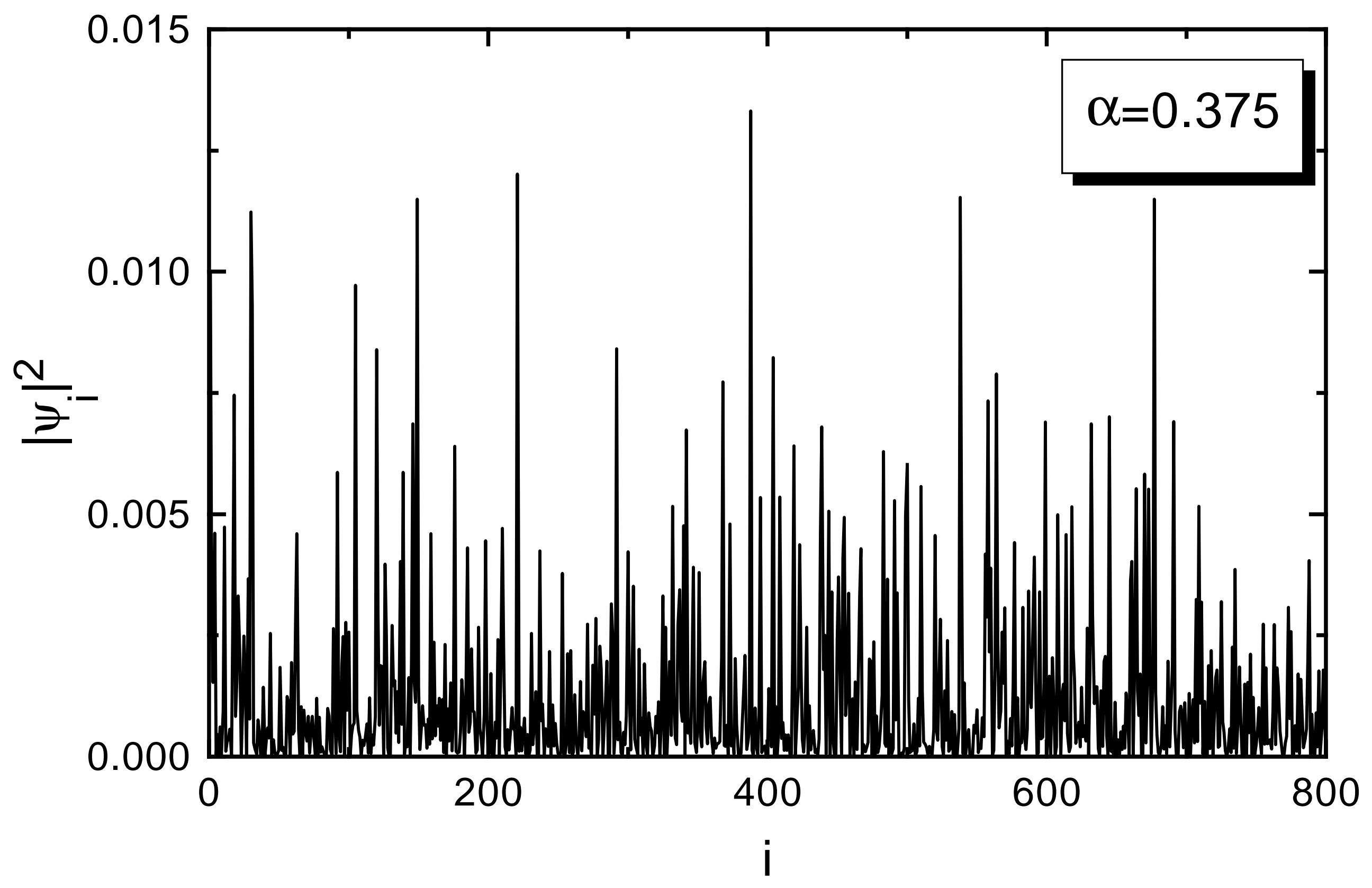




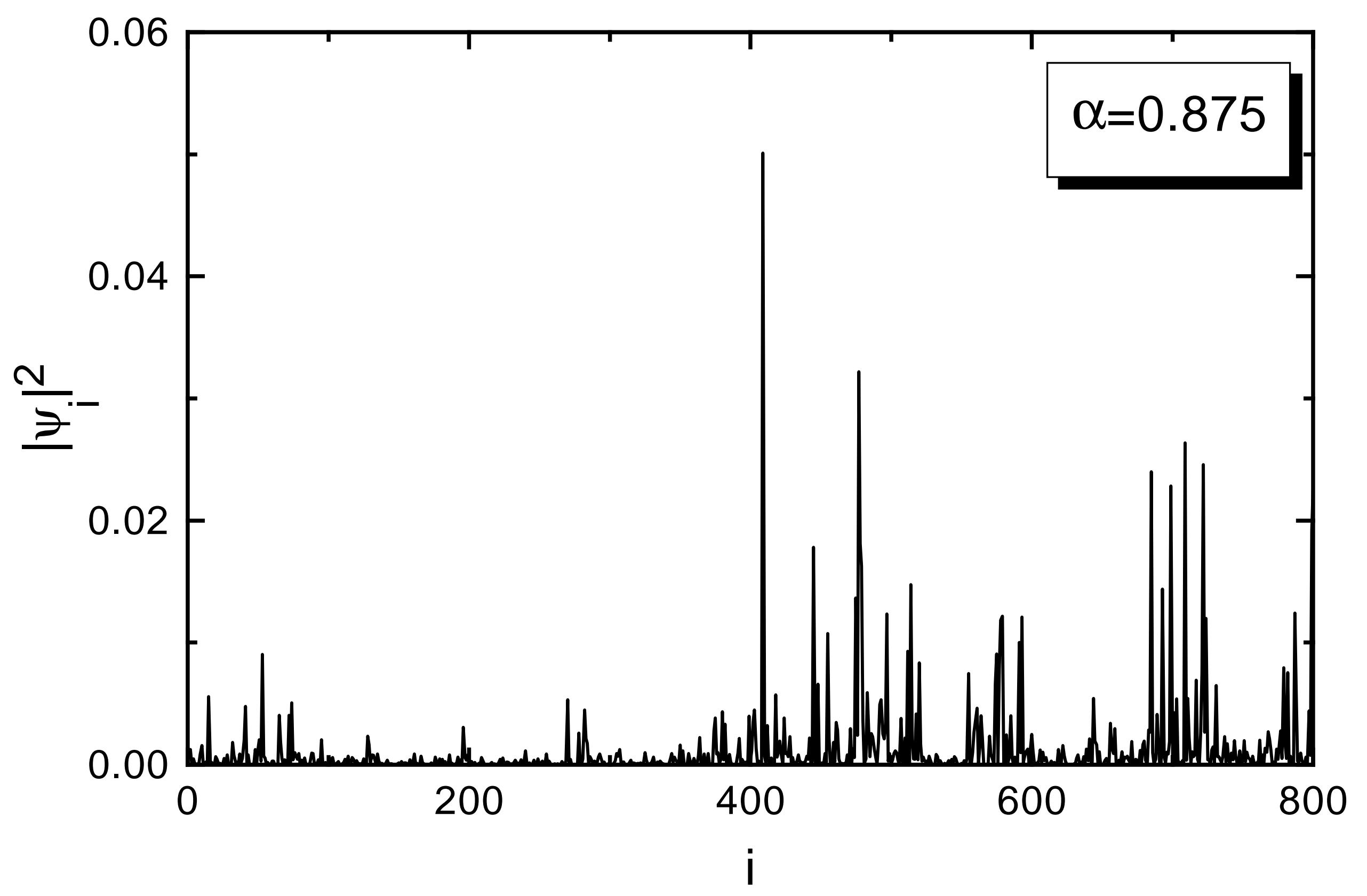









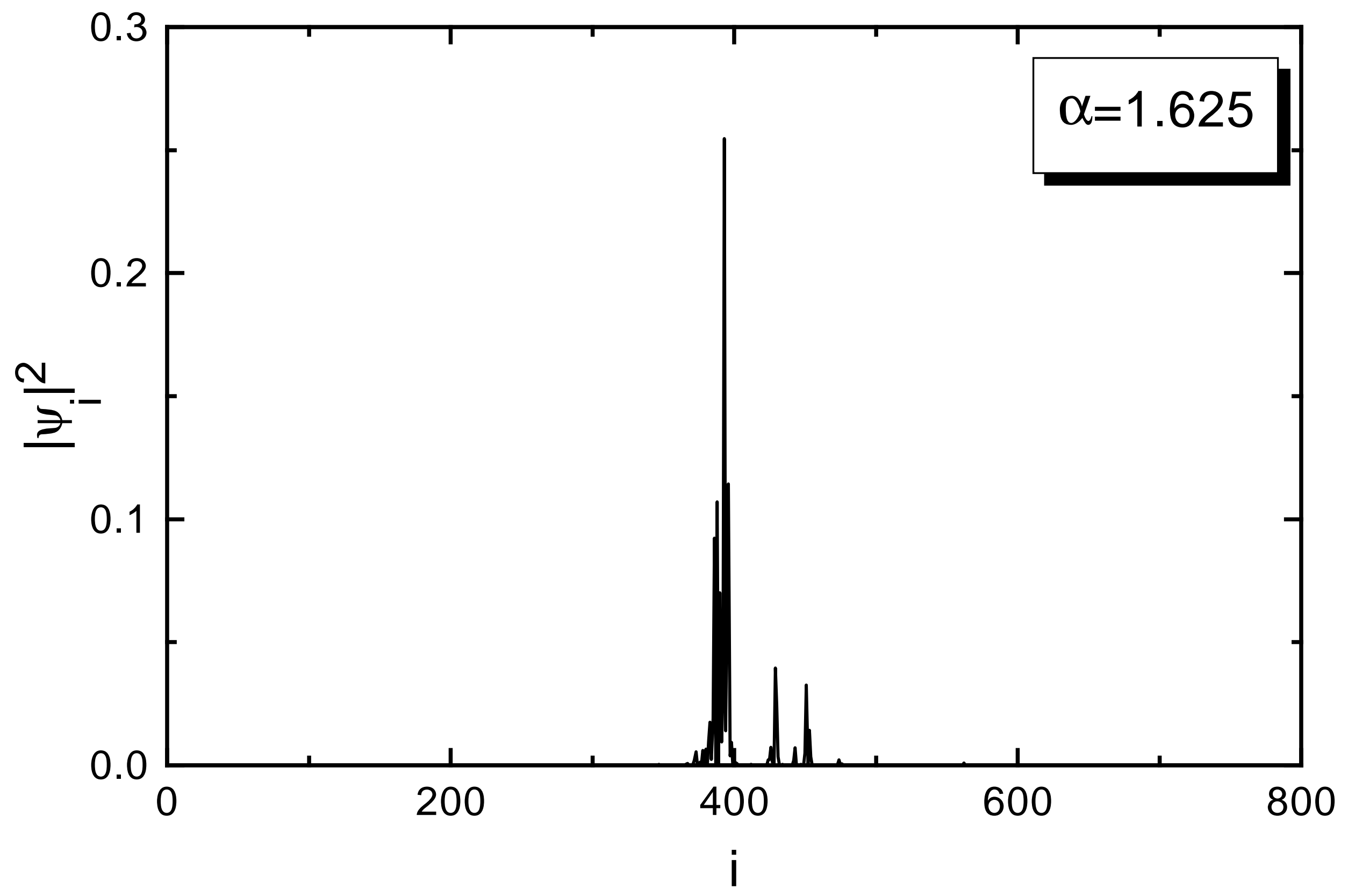









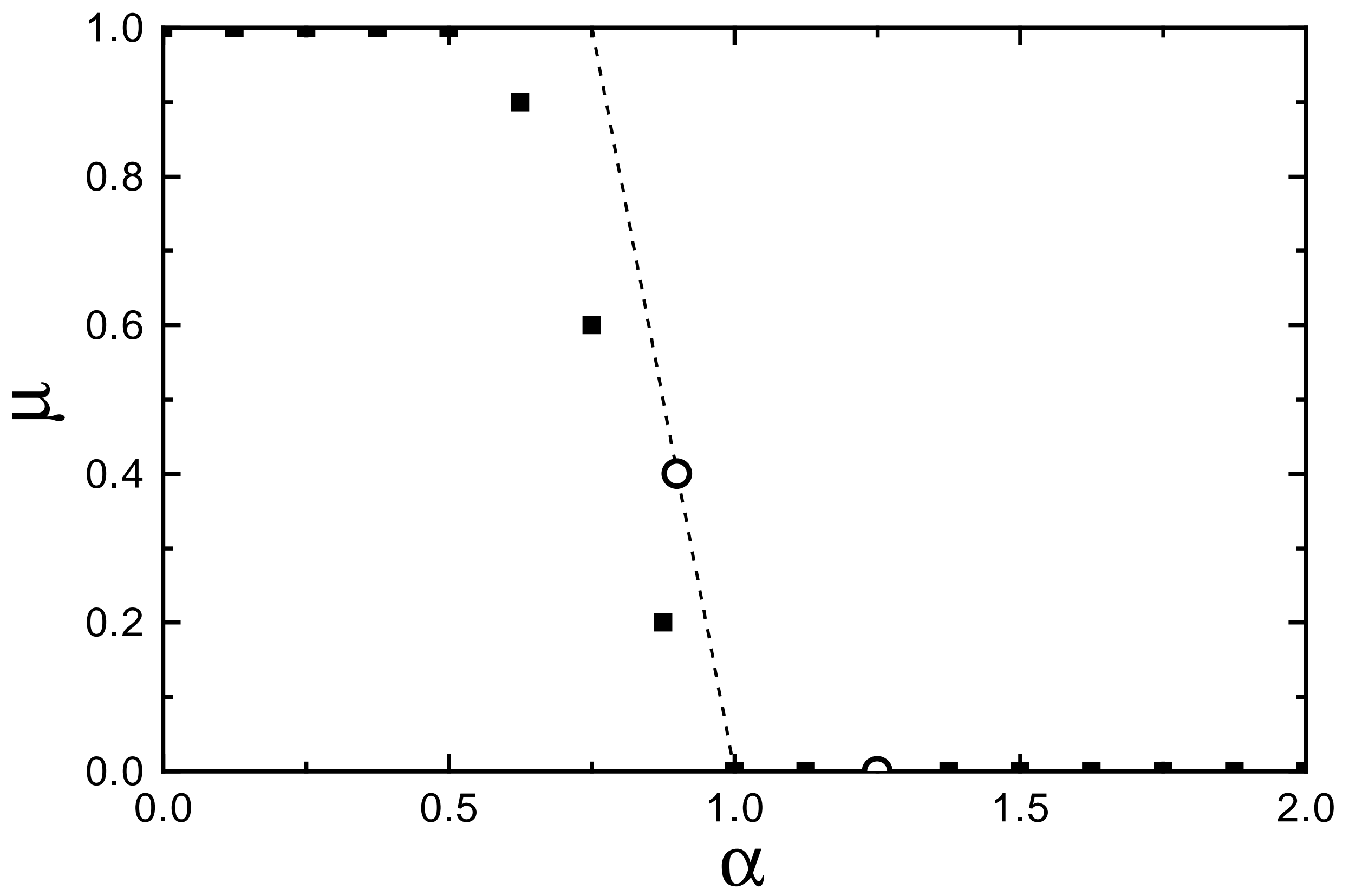

